\title{
Development and validation of a nomogram to predict postoperative pulmonary complications following thoracoscopic surgery
}

\author{
Bin Wang ${ }^{\text {Equal first author, } 1}{ }^{1}$, Zhenxing Chen ${ }^{\text {Equal first author, } 1}{ }^{1}$, Ru Zhao ${ }^{1}$, Li Zhang ${ }^{1}$, Ye Zhang ${ }^{\text {Corresp. } 1}$ \\ ${ }^{1}$ Department of Anesthesiology and Perioperative Medicine, The Second Affiliated Hospital of Anhui Medical University, Hefei, China \\ Corresponding Author: Ye Zhang \\ Email address: zhangye_hassan@sina.com
}

Abstract Background: Postoperative pulmonary complications (PPCs) after thoracoscopic surgery are common. This retrospective study aimed to develop a nomogram to predict PPCs in thoracoscopic surgery . Methods: A total of 905 patients who underwent thoracoscopy were randomly enrolled and divided into a training cohort and a validation cohort at $80 \%: 20 \%$. The training cohort was used to develop a nomogram model, and the validation cohort was used to validate the model. Univariate and multivariable logistic regression were applied to screen risk factors for PPCs, and the nomogram was incorporated in the training cohort. The discriminative ability and calibration of the nomogram for predicting PPCs were assessed using C-indices and calibration plots.

Results: Among the patients, 207 (22.87\%) presented PPCs, including 166 cases in the training cohort and 41 cases in the validation cohort. Using backward stepwise selection of clinically important variables with the Akaike information criterion (AIC) in the training cohort, the following seven variables were incorporated for predicting PPCs: American Society of Anesthesiologists (ASA) grade III/IV, operation time longer than 180 min, onelung ventilation time longer than $60 \mathrm{~min}$, and history of stroke, heart disease, chronic obstructive pulmonary disease (COPD) and smoking. With incorporation of these factors, the nomogram achieved good $\mathrm{C}$-indices of 0.894 [95\% confidence interval $(\mathrm{Cl})$, $0.866-0.921]$ and $0.868(95 \% \mathrm{Cl}, 0.811-0.925)$ in the training and validation cohorts, respectively, with well-fitted calibration curves. Conclusion: The nomogram offers good predictive performance for PPCs after thoracoscopic surgery. This model may help distinguish the risk of PPCs and make reasonable treatment choices. 
1 Development and validation of a nomogram to predict postoperative pulmonary complications

2 following thoracoscopic surgery

3 Bin Wang, $\mathrm{MD}^{1}$, Zhenxing Chen, $\mathrm{MS}^{1}$, Ru Zhao, MS, Li Zhang, PhD, Ye Zhang, MD, PhD*

4 Department of Anesthesiology and Perioperative Medicine, The Second Affiliated Hospital of

5 Anhui Medical University, Hefei, China

6

$7 \quad{ }^{1}$ These authors contributed equally to this work.

9 Corresponding Author: Ye Zhang, MD, PhD, Department of Anesthesiology and Perioperative

10 Medicine, The Second Affiliated Hospital of Anhui Medical University, 678\# Furong Road, Hefei,

11 Anhui Province, China

12 E-mail address: zhangye_hassan@sina.com

13 E-mail addresses:

14 B. Wang,w1986wb@163.com

15 Z. Chen, chenzhiyudhd@sina.com

R. Zhao, zhaoruru13@163.com

L. Zhang, 504306585@qq.com 
Abstract

Background: Postoperative pulmonary complications (PPCs) after thoracoscopic surgery are common. This retrospective study aimed to develop a nomogram to predict PPCs in thoracoscopic surgery.

Methods: A total of 905 patients who underwent thoracoscopy were randomly enrolled and divided into a training cohort and a validation cohort at $80 \%: 20 \%$. The training cohort was used to develop a nomogram model, and the validation cohort was used to validate the model. Univariate and multivariable logistic regression were applied to screen risk factors for PPCs, and the nomogram was incorporated in the training cohort. The discriminative ability and calibration of the nomogram for predicting PPCs were assessed using C-indices and calibration plots.

Results: Among the patients, 207 (22.87\%) presented PPCs, including 166 cases in the training cohort and 41 cases in the validation cohort. Using backward stepwise selection of clinically

31 important variables with the Akaike information criterion (AIC) in the training cohort, the

32 following seven variables were incorporated for predicting PPCs: American Society of Anesthesiologists (ASA) grade III/IV, operation time longer than $180 \mathrm{~min}$, one-lung ventilation time longer than 60 min, and history of stroke, heart disease, chronic obstructive pulmonary disease (COPD) and smoking. With incorporation of these factors, the nomogram achieved good C-indices of 0.894 [95\% confidence interval $(\mathrm{Cl}), 0.866-0.921$ ] and $0.868(95 \% \mathrm{Cl}, 0.811-0.925)$ in the training and validation cohorts, respectively, with well-fitted calibration curves.

Conclusion: The nomogram offers good predictive performance for PPCs after thoracoscopic 
39 surgery. This model may help distinguish the risk of PPCs and make reasonable treatment

40 choices.

41 Clinical Trial Registration: Chinese Clinical Trial Registry (No. ChiCTR2000033329), date of

42 registration May 28, 2020, http://www.chictr.org.cn/showproj.aspx?proj=54264.

43 Keywords: Postoperative pulmonary complications; Thoracoscopic surgery; Nomograms 


\section{Background}

Postoperative pulmonary complications (PPCs) are very common in thoracic surgery and are major causes of increased morbidity, mortality, and prolonged hospital stay during the perioperative period (Ball et al. 2018; Haller \& Walder 2017; Hayami et al. 2018; Li et al. 2020; Mathis et al. 2019; Moore et al. 2017; Sayal et al. 2018). Thoracic surgery tends to involve onelung ventilation, long operation times, increased trauma, sternum or rib incision, nerve injury, and acute or chronic pain. As these characteristics lead to many complications and seriously affect the prognosis of patients, PPCs of thoracic surgery have been widely studied by surgeons and anesthesiologists (Hoshikawa \& Tochii 2017; Khaitan \& D'Amico 2018; Medbery \& Force 2017; Munoz de Cabo et al. 2020). The incidence of PPCs after thoracic surgery is $19-59 \%$ (Agostini et al. 2010; Garcia-Miguel et al. 2003).

Thoracoscopic surgery reduces the risk of PPCs (Cypel \& Yasufuku 2018; Wong et al. 2018). Some studies have found that compared to thoracotomy, thoracoscopic surgery significantly reduces the incidence of PPCs. Nevertheless, the incidence of PPCs after thoracoscopic surgery is still as high as $16-30 \%$ due to the characteristics of the surgery, such as the need for one-lung ventilation and lung or diaphragm surgery (Kaufmann et al. 2019; Nozaki et al. 2018). age, cardiovascular complications, smoking, and chronic obstructive pulmonary disease (COPD) (Licker et al. 2006; Stephan et al. 2000). Although independent risk factors for PPCs in thoracic surgery have been examined, a simple and effective prediction model has not yet been 
established, and specific predictive studies on PPCs after thoracoscopic surgery have not been carried out.

The present study aimed to identify risk factors for PPCs of thoracoscopic surgery based on demographic and clinical data of patients and to establish a nomogram for predicting PPCs through independent risk factors that could serve as a reference for clinical practice.

\section{Methods}

\section{Study population}

This retrospective study was approved by the Ethics Committee of the Second Affiliated Hospital of Anhui Medical University (No. YX2020-038) and registered in the Chinese Clinical Trial Registry (No. ChiCTR2000033329), in accordance with the TRIPOD checklist. From August 2018 to March 2020, 905 patients who underwent thoracoscopic surgery at the Second Affiliated Hospital of Anhui Medical University were enrolled and analyzed. The following were exclusion criteria: emergency and trauma patients; age < 18 years old; patients with pulmonary infection and pleural effusion before the operation; patients who underwent thoracotomy or if the operation was cancelled; patients who underwent re-operations; and patients whose relevant data were missing or incomplete. 
95

according to the following methods: A. If the patient has passed away or cannot be contacted, apply for exemption of informed consent according to the process of ethics committee; B. If patients agree to include their medical records in this study, they should sign the informed consent; C. If the patient agrees to provide his/her data for this study while does not want to sign the informed consent form, a witness must be present. The data can only be included after the witness signed in the informed consent; D. If patients explicitly refuse to include their medical records in this study, their data will be excluded.

\section{Data collection}

Based on clinical importance, scientific knowledge, previous research (Agostini et al. 2010; Licker et al. 2006; Stephan et al. 2000) and the actual data that could be collected for a retrospective study, 27 demographic and clinical variables of patients undergoing thoracoscopic surgery were collected. General information included age, sex, American Society of Anesthesiologists (ASA) grade, preoperative mean arterial pressure (MAP), body mass index (BMI), and history of hypertension, diabetes mellitus, stroke, heart disease, COPD, smoking (ever or current), and alcohol use. Laboratory examination data before the operation included leukocyte, red blood cell, and platelet counts and aspartate aminotransferase, alanine aminotransferase, blood glucose, serum creatinine, blood urea nitrogen, serum sodium, serum potassium, and serum albumin levels. Information on the operation and anesthesia management included the duration of the operation, airway management, the duration of onelung ventilation, and tpyes of operation. 
115 The primary end-point of this study was PPCs, which were defined according to the

116 European Perioperative Clinical Outcome (EPCO), including respiratory infection, respiratory

117 failure, pleural effusion, atelectasis, pneumothorax, bronchospasm, and aspiration pneumonia

118 (Jammer et al. 2015; Miskovic \& Lumb 2017) (Supplementary Table 1). An event was considered

119 a PPC if one of these complications was detected within the first 7 postoperative days. The

120 diagnosis of PPCs was made by two experienced researchers based on the patient's clinical data.

121 In the case of a dispute, the diagnosis was discussed by the research group.

122

123

124

125

126

127

128

129

130

131

132

133

134

\section{Statistical analysis}

All patient demographic and clinical data were randomly divided into a training cohort and validation cohort according to an $80 \%: 20 \%$ ratio by setting the random seed " 1234567 " in the "caTools" package of R software (version 3.6.3; http://www.r-project.org). The training and validation cohorts were used to derive and validate the model, respectively. Continuous variables such as age and BMI were categorized after being assessed based on clinical reference values and scientific knowledge. The data were compared using the $\chi^{2}$ test or Fisher's exact test, and the results are expressed as whole numbers and proportions.

Univariate logistic regression analysis was performed to identify risk factors for PPCs following thoracoscopic surgery in the training cohort. All variables associated with PPCs with a $p$-value less than 0.1 were candidates for backward stepwise multivariate analysis with the Akaike information criterion (AIC) to investigate independent risk factors. The nomogram was formulated based on the results of AIC multivariate logistic regression analysis in the training 
135 cohort, and selected variables were incorporated in the nomogram to predict the probability of 136 PPCs.

The total scores of the nomogram were calculated for each patient in the training cohort. A receiver operating characteristic (ROC) curve was analyzed to calculate the optimal cut-off points based on the Youden index in the training cohort, after which the cut-off points in the training cohort were used in both cohorts to verify the generalizability of the model. The accuracy, precision, sensitivity, specificity, and the concordance index (C-index) were calculated, and ROC curves were constructed for the training and validation cohorts. The predictive performance of the nomogram was assessed by the C-index with 1000 bootstrap replicates and calibrated with 100 bootstrap replicates to decrease overfitting in the training and validation cohorts, respectively. A C-index value of 0.5 indicates no discrimination; a value of 1.0 indicates perfect discrimination ability with different outcomes. In an excellently calibrated model, the predictions should fall on a diagonal line of 45 degrees, which represents the ideal relationship between the observed outcome frequencies and the predicted probabilities.

All tests were 2 -tailed, and $p$-values less than 0.05 were considered statistically significant. Statistical analysis was carried out using SPSS 22.0 software (Chicago, IL, USA) and R software. The R software packages included rms, foreign, caTools, tableone and pROC.

\section{Results}


155

156

157

158

159

160

161

162

163

164

165

166

167

168

169

170

171

172

173

174

175

\section{Patient characteristics}

During the study period, 1084 consecutive patients underwent thoracoscopic surgery. Among the cases, 48 involved emergency and trauma surgery, 13 patients were younger than 18 years old, 21 cases were converted to open surgery, and there were missing or incomplete data for 97 patients. No patient explicitly refused to include his/her data for this research. Thus, a total of 905 patients who met the inclusion criteria were included in the present study. All patient demographic and clinical data were randomly divided into a training cohort (724 cases) and a validation cohort (181 cases) at a ratio of 80\%:20\% (Supplementary Figure 1). Of the included cases, 207 patients exhibited PPCs, with an incidence of $22.87 \%$. The details of the PPCs are shown in Supplementary Table 2. Among those who experienced PPCs, 166 patients (22.93\%) were allocated to the training cohort, and 41 patients $(22.65 \%)$ were allotted to the validation cohort. The demographic and clinical data of the training and validation cohorts are shown in

Table 1. The $p$-values and standardized mean differences (SMDs) suggested that the partition of training cohort data and validation cohort data was balanced.

\section{Independent risk factors for PPCs}

Backward stepwise selection using the AIC in logistic regression modelling identified the following 7 variables as having the strongest associations with PPC risk: ASA grade III/IV, operation time longer than $180 \mathrm{~min}$, one-lung ventilation time longer than $60 \mathrm{~min}$, and history of stroke, heart disease, COPD, and smoking. According to the multivariable analysis, ASA grade III/IV [odds ratio (OR), 10.171; 95\% confidence interval (CI), 5.598-18.926; $p<0.001$ ], operation time longer than $180 \min (\mathrm{OR}, 2.480 ; 95 \% \mathrm{Cl}, 1.363-4.563 ; p=0.003)$ or longer than $300 \mathrm{~min}(\mathrm{OR}$, 
176

177

178

179

180

181

182

183

184

185

186

187

188

189

190

191

192

193

194

195

196

12.516; $95 \% \mathrm{Cl}, 5.232-17.766 ; p<0.001)$, one-lung ventilation time longer than 60 min (OR, 2.947; $95 \% \mathrm{Cl}, 1.257-7.648 ; p=0.018)$, history of stroke (OR, 4.209; 95\% $\mathrm{Cl}, 1.518-11.683 ; p=$ 0.006), history of COPD (OR, 2.888; $95 \% \mathrm{Cl}, 1.230-6.980 ; p=0.016)$, and history of smoking (OR, 4.101; 95\% Cl, 2.544-6.654; $p<0.001$ ) were each independently associated with PPCs (Table 2).

\section{Development and validation of a PPC-predicting nomogram}

The nomogram acquired from the training cohort to predict PPCs after thoracoscopic surgery is shown in Figure 1. The nomogram was created based on the following 7 factors: ASA grade (I/II or III/IV), operation time $(<180,180-300$, or $\geq 300 \mathrm{~min})$, one-lung ventilation time $(<$ 60 or $\geq 60 \mathrm{~min}$ ), history of stroke (no or yes), history of heart disease (no or yes), history of COPD (no or yes), and history of smoking (no or yes). A higher point total based on the sum of the assigned number of points for each factor in the nomogram was associated with a higher risk of PPCs.

The total scores of the nomogram of each patient in the training cohort were calculated. ROC curves were analyzed to calculate the optimal cut-off point based on the Youden index in the training cohort, which yielded a value of 134 points. The accuracy, precision, sensitivity, specificity, and C-index are presented in Table 3. This cut-off point was used in both cohorts. The areas under the ROC curve (AUCs) were $0.894(95 \% \mathrm{Cl}, 0.866-0.922)$ and $0.867(95 \% \mathrm{Cl} 0.810-$ 0.925) in the training and validation cohorts, respectively (Figure 2). In addition, the C-indices with 1000 bootstrap replicates were $0.894(95 \% \mathrm{Cl}, 0.866-0.921)$ and $0.868(0.811-0.925)$ in the training and validation cohorts, respectively (Table 2), indicating the good performance of the nomogram in estimating the risk of PPCs. The calibration plots with 100 bootstrap results of the 
197 training and validation cohorts are shown in Figure 3, which vividly demonstrates good

198 agreement regarding the presence of PPCs between the risk estimation by the nomogram

199 acquired from the training cohort and the actual PPC occurrence in the training and validation

200 cohorts.

201

202

203

\section{Discussion}

204

In the current study, a nomogram was developed to predict the risk of PPCs after

thoracoscopic surgery. This nomogram was constructed using the training cohort and achieved good performance in both the training and validation cohorts; the ROC and C-index results were also good in both cohorts. Nomogram is a very useful tool to predict diseases or outcomes (Gu constructed in this study had good predictive performance and generalizability. Overall, building a prediction model for PPCs after thoracoscopic surgery is important for the perioperative management of patients, and the model can help with early intervention for patient complications, thus improving the quality of surgery and reducing medical costs. Moreover, obtaining information about patient risk factors, such as medical history or routine preoperative examinations, is conducive to appropriate clinical practice and implementation.

In this study, the incidence of PPCs during thoracoscopic surgery was $22.9 \%$. In general, thoracic surgery has a high incidence of PPCs. Although thoracoscopic surgery reduces the 
217 incidence, PPCs are still common. The incidence of PPCs in this study was consistent with previous

218 research results (Kaufmann et al. 2019; Nozaki et al. 2018) but higher than that in some studies

219 (Agostini et al. 2017; Ceppa et al. 2012). In the present study, the diagnostic criteria for PPCs were based on EPCO guidelines (Jammer et al. 2015; Miskovic \& Lumb 2017), which were published in 2015 to standardize the definition of PPCs, as these are composite outcome indicators and exhibit certain differences in clinical evaluation criteria. Respiratory infection, respiratory failure, pleural effusion, atelectasis, pneumothorax, bronchospasm, and aspiration pneumonia are included in the guidelines that define PPCs. However, the incidence of PPCs in this study was somewhat high because of the broad definition of PPCs in the guidelines, e.g., pure pleural effusion is also listed as a pulmonary complication. model easy to extend and apply, and seven indicators were included: ASA grade, duration of operation time, duration of one-lung ventilation, and history of stroke, heart disease, COPD, and smoking. Nomograms are simple and practical and can be widely used in clinical practice with the nomogram, only one variable should be positioned on the corresponding axis, and the points research in the field of anesthesia and achieved good results (Ferrando et al. 2020; Ge et al. 2018; 
237 independent risk factors were similar to those in a previous study. Indeed, the reported risk

238 factors for pulmonary complications in thoracic surgery include older age, cardiovascular 239 complications, smoking, COPD history, and high BMI and ASA grade (Agostini et al. 2010; Licker

240 et al. 2006; Stephan et al. 2000). By using backward stepwise selection with the AIC in logistic

241 regression modelling, we identified the following 7 variables as having the strongest associations

242 with PPC risk: ASA grade III/IV, operation time longer than $180 \mathrm{~min}$, one-lung ventilation time

243 longer than $60 \mathrm{~min}$, and history of stroke, heart disease, COPD, and smoking. Conversely, some

244 indicators, such as older age, were not included as predictors of risk. In the univariate logistic regression, age differed significantly, but it was not an independent risk factor in the multivariate regression. The reason for this finding may be that elderly patients with other diseases, such as stroke, COPD, and heart disease, in our study population had higher ASA grades. Nonetheless, older age and these factors have obvious collinearity. the total points obtained from each indicator in the nomogram means greater the possibility of PPCs. Because of the convenience to obtain the indicators in clinical work, so it is convenient to evaluate whether patients are prone to postoperative complications according to the nomogram, so as to early identify high-risk patients, and early detect, intervene, and improve postoperative outcomes of patients. For example, an ASA grade III patient undergoing thoracoscopic surgery 
257 of 293 points for PPCs (100 points for ASA grade III, 39 points for duration of operation, 47 points

258 for duration of one-lung ventilation, 0 points for history of stroke, 0 points for history of heart

259 disease, 46 points for history of COPD, and 61 points for history of smoking). The predicted PPCS

260 risk of this patient was about $92 \%$.

261 The present study has some limitations. This was a retrospective study, and PPCs

262 may be missed or misdiagnosed. The case data may not fully reflect the PPCs and diseases of

263 patients, and some data could not be obtained. Moreover, this was a single-center study with a

264 small sample size, and all thoracoscopic surgeries were included. Some variables, such as blood

265 transfusion, could not be included in the model because of too few transfusion patients and large

266 random errors. PPCs have a relatively broad and general definition, and the degree of specific

267 complications was not analyzed. Multicenter, large-sample, prospective studies and subgroup 268 analyses should be carried out in the future.

Conclusions

A nomogram was constructed by combining seven risk factors for PPCs. The model provides

a good estimation of PPC risk in patients after thoracoscopic surgery, which may be useful for patients who undergo thoracoscopic surgery. 
276

PPC: Postoperative pulmonary complication; AIC: Akaike information criterion; ASA: American

Society of Anesthesiologists; COPD: chronic obstructive pulmonary disease; $\mathrm{Cl}$ : confidence interval; BMI: body mass index; AIDS: acquired immunodeficiency syndrome; MAP: mean arterial pressure; EPCO: European Perioperative Clinical Outcome; ROC: receiver operating characteristic; SMD: standardized mean difference; OR: odds ratio; AUC: area under the ROC curve; LMA: laryngeal mask airway; SD: standard deviation

\section{Acknowledgements:}

We thank Dr Hui Peng and Dr Lin Sun for their help with the statistical analysis. We also thank the patients who participated in this study.

\section{Funding:}

This study was supported by the Department of Science and Technology of Anhui Province, Hefei, China (No. 1704a0802165) and the Natural Science Foundation of Anhui Province (No. 1908085QH358).

\section{Availability of data and materials:}

The datasets used and analyzed during the current study are available from the corresponding author on reasonable request.

\section{Ethics approval:}


294 This study was approved by the Institutional Review Board and Hospital Research Ethics

295 Committee of The Second Affiliated Hospital of Anhui Medical University [No. YX2020-038].

296

297

298

299

300

301

302

303

304

305

306

307

308

309

310

311

312

313

314

\section{Consent for publication:}

Not applicable.

Competing interests:

The authors declare that they have no competing interests.

\section{REFERENCES}

Agostini P, Cieslik H, Rathinam S, Bishay E, Kalkat MS, Rajesh PB, Steyn RS, Singh S, and Naidu B. 2010. Postoperative pulmonary complications following thoracic surgery: are there any modifiable risk factors? Thorax 65:815-818. 10.1136/thx.2009.123083

Agostini P, Lugg ST, Adams K, Vartsaba N, Kalkat MS, Rajesh PB, Steyn RS, Naidu B, Rushton A, and Bishay E. 2017. Postoperative pulmonary complications and rehabilitation requirements following lobectomy: a propensity score matched study of patients undergoing video-assisted thoracoscopic surgery versus thoracotomydagger. Interact Cardiovasc Thorac Surg 24:931-937. 10.1093/icvts/ivx002

Ball L, Hemmes SNT, Serpa Neto A, Bluth T, Canet J, Hiesmayr M, Hollmann MW, Mills GH, Vidal Melo MF, Putensen C, Schmid W, Severgnini P, Wrigge H, Gama de Abreu M, Schultz MJ, 
Pelosi P, investigators LV, Network P, and Clinical Trial Network of the European Society of A. 2018. Intraoperative ventilation settings and their associations with postoperative pulmonary complications in obese patients. $\mathrm{Br} J$ Anaesth 121:899-908. 10.1016/j.bja.2018.04.021

Ceppa DP, Kosinski AS, Berry MF, Tong BC, Harpole DH, Mitchell JD, D'Amico TA, and Onaitis MW. 2012. Thoracoscopic lobectomy has increasing benefit in patients with poor pulmonary function: a Society of Thoracic Surgeons Database analysis. Ann Surg 256:487493. $10.1097 /$ SLA.0b013e318265819c

Cypel M, and Yasufuku K. 2018. Complications during minimal invasive thoracic surgery: are new surgeons prepared? Lancet Oncol 19:17-19. 10.1016/S1470-2045(17)30915-30914

Ferrando C, Suarez-Sipmann F, Librero J, Pozo N, Soro M, Unzueta C, Brunelli A, Peiro S, Llombart A, Balust J, Aldecoa C, Diaz-Cambronero O, Franco T, Redondo FJ, Garutti I, Garcia JI, Ibanez M, Granell M, Rodriguez A, Gallego L, de la Matta M, Marcos JM, Garcia J, Mazzinari G, Tusman G, Villar J, Belda J, and Individualized PeRioperative Openlung VN. 2020. A noninvasive postoperative clinical score to identify patients at risk for postoperative pulmonary complications: the Air-Test Score. Minerva Anestesiol 86:404415. 10.23736/S0375-9393.19.13932-6

Garcia-Miguel FJ, Serrano-Aguilar PG, and Lopez-Bastida J. 2003. Preoperative assessment. Lancet 362:1749-1757. 10.1016/s0140-6736(03)14857-x

Ge H, Jiang Y, Jin Q, Wan L, Qian X, and Zhang Z. 2018. Nomogram for the prediction of postoperative hypoxemia in patients with acute aortic dissection. BMC Anesthesiol 18:146. 10.1186/s12871-018-0612-7

Gu W, Hu M, Wang W, Shi C, and Mei J. 2020. Development and Validation of a Novel Nomogram for Predicting Tumor-Distant-Metastasis in Patients with Early T1-2 Stage Lung Adenocarcinoma. Ther Clin Risk Manag 16:1213-1225. 10.2147/TCRM.S272748

Hahn RG. 1995. Life-threatening transurethral resection syndrome despite monitoring of fluid absorption with ethanol. Eur J Anaesthesiol 12:431-433. 
342 Haller G, and Walder B. 2017. Postoperative pulmonary complications - Still room for improvement. Eur J Anaesthesiol 34:489-491. 10.1097/EJA.0000000000000659

344

345

346

347

348

Hayami M, Watanabe M, Ishizuka N, Mine S, Imamura Y, Okamura A, Kurogochi T, and Yamashita K. 2018. Prognostic impact of postoperative pulmonary complications following salvage esophagectomy after definitive chemoradiotherapy. J Surg Oncol 117:1251-1259. 10.1002/jso.24941

Hoshikawa Y, and Tochii D. 2017. Postoperative Atelectasis and Pneumonia after General Thoracic Surgery. Kyobu Geka 70:649-655.

Jammer I, Wickboldt N, Sander M, Smith A, Schultz MJ, Pelosi P, Leva B, Rhodes A, Hoeft A, Walder B, Chew MS, Pearse RM, European Society of A, the European Society of Intensive Care M, European Society of A, and European Society of Intensive Care M. 2015. Standards for definitions and use of outcome measures for clinical effectiveness research in perioperative medicine: European Perioperative Clinical Outcome (EPCO) definitions: a statement from the ESA-ESICM joint taskforce on perioperative outcome measures. Eur J Anaesthesiol 32:88-105. 10.1097/EJA.0000000000000118

Jung W, Park T, Kim Y, Park H, Han Y, He J, Wolfgang CL, Blair A, Rashid MF, Kluger MD, Su GH, Chabot JA, Yang CY, Lou W, Valente R, Del Chiaro M, Shyr YM, Wang SE, van Huijgevoort NCM, Besselink MG, Yang Y, Kim H, Kwon W, Kim SW, and Jang JY. 2019. Validation of a nomogram to predict the risk of cancer in patients with intraductal papillary mucinous neoplasm and main duct dilatation of $10 \mathrm{~mm}$ or less. $\mathrm{Br} \mathrm{J}$ Surg 106:1829-1836. 10.1002/bjs.11293

Kaufmann KB, Loop T, Heinrich S, and Working Group of the German Thorax R. 2019. Risk factors for post-operative pulmonary complications in lung cancer patients after videoassisted thoracoscopic lung resection: Results of the German Thorax Registry. Acta Anaesthesiol Scand 63:1009-1018. 10.1111/aas.13388

Khaitan PG, and D'Amico TA. 2018. Milestones in thoracic surgery. J Thorac Cardiovasc Surg 155:2779-2789. 10.1016/j.jtcvs.2017.12.149 
Kong J, Wang T, Shen S, Zhang Z, Yang X, and Wang W. 2019. A genomic-clinical nomogram predicting recurrence-free survival for patients diagnosed with hepatocellular carcinoma. PeerJ 7:e7942. 10.7717/peerj.7942

Lei Z, Li J, Wu D, Xia Y, Wang Q, Si A, Wang K, Wan X, Lau WY, Wu M, and Shen F. 2016. Nomogram for Preoperative Estimation of Microvascular Invasion Risk in Hepatitis B Virus-Related Hepatocellular Carcinoma Within the Milan Criteria. JAMA Surg 151:356363. 10.1001/jamasurg.2015.4257

Li X, and Meng Y. 2019. A prognostic nomogram for neuroblastoma in children. PeerJ 7:e7316. 10.7717/peerj.7316

Li XF, Jiang D, Jiang YL, Yu H, Zhang MQ, Jiang JL, He LL, and Yu H. 2020. Comparison of low and high inspiratory oxygen fraction added to lung-protective ventilation on postoperative pulmonary complications after abdominal surgery: A randomized controlled trial. J Clin Anesth 67:110009. 10.1016/j.jclinane.2020.110009

Licker MJ, Widikker I, Robert J, Frey JG, Spiliopoulos A, Ellenberger C, Schweizer A, and Tschopp JM. 2006. Operative mortality and respiratory complications after lung resection for cancer: impact of chronic obstructive pulmonary disease and time trends. Ann Thorac Surg 81:1830-1837. 10.1016/j.athoracsur.2005.11.048

Lu C, Yu L, Wei J, Chen J, Zhuang J, and Wang S. 2019. Predictors of postoperative outcomes in infants with low birth weight undergoing congenital heart surgery: a retrospective observational study. Ther Clin Risk Manag 15:851-860. 10.2147/TCRM.S206147

Mathis MR, Duggal NM, Likosky DS, Haft JW, Douville NJ, Vaughn MT, Maile MD, Blank RS, Colquhoun DA, Strobel RJ, Janda AM, Zhang M, Kheterpal S, and Engoren MC. 2019. Intraoperative Mechanical Ventilation and Postoperative Pulmonary Complications after Cardiac Surgery. Anesthesiology 131:1046-1062. 10.1097/ALN.0000000000002909

Medbery RL, and Force SD. 2017. Quality and Cost in Thoracic Surgery. Thorac Surg Clin 27:267277. 10.1016/j.thorsurg.2017.03.006

Miskovic A, and Lumb AB. 2017. Postoperative pulmonary complications. Br J Anaesth 118:317- 
396

397

398

399

400

401

402

403

404

405

406

407

408

409

410

411

412

413

414

415

416

417

418

419

420

421

422

334. $10.1093 / \mathrm{bja} / \mathrm{aex} 002$

Moore JA, Conway DH, Thomas N, Cummings D, and Atkinson D. 2017. Impact of a perioperative quality improvement programme on postoperative pulmonary complications. Anaesthesia 72:317-327. 10.1111/anae.13763

Munoz de Cabo C, Hermoso Alarza F, Cossio Rodriguez AM, and Martin Delgado MC. 2020. Perioperative management in thoracic surgery. Med Intensiva 44:185-191. 10.1016/j.medin.2019.10.012

Nozaki I, Mizusawa J, Kato K, Igaki H, Ito Y, Daiko H, Yano M, Udagawa H, Nakagawa S, Takagi M, and Kitagawa Y. 2018. Impact of laparoscopy on the prevention of pulmonary complications after thoracoscopic esophagectomy using data from JCOG0502: a prospective multicenter study. Surg Endosc 32:651-659. 10.1007/s00464-017-5716-5

Sayal P, Bateman BT, Menendez M, Eikermann M, and Ladha KS. 2018. Opioid Use Disorders and the Risk of Postoperative Pulmonary Complications. Anesth Analg 127:767-774. 10.1213/ANE.0000000000003307

Stephan F, Boucheseiche S, Hollande J, Flahault A, Cheffi A, Bazelly B, and Bonnet F. 2000. Pulmonary complications following lung resection: a comprehensive analysis of incidence and possible risk factors. Chest 118:1263-1270. 10.1378/chest.118.5.1263

Wong MKH, Sit AKY, and Au TWK. 2018. Minimally invasive thoracic surgery: beyond surgical access. J Thorac Dis 10:S1884-S1891. 10.21037/jtd.2018.05.196

Yap WK, Shih MC, Kuo C, Pai PC, Chou WC, Chang KP, Tsai MH, and Tsang NM. 2018. Development and Validation of a Nomogram for Assessing Survival in Patients With Metastatic Lung Cancer Referred for Radiotherapy for Bone Metastases. JAMA Netw Open 1:e183242. 10.1001/jamanetworkopen.2018.3242

Yuan K, Chen J, Xu P, Zhang X, Gong X, Wu M, Xie Y, Wang H, Xu G, and Liu X. 2020. A Nomogram for Predicting Stroke Recurrence Among Young Adults. Stroke 51:1865-1867. 10.1161/STROKEAHA.120.029740

Zhou XD, Dong WH, Zhao CH, Feng XF, Wen WW, Tu WY, Cai MX, Xu TC, and Xie QL. 2019. Risk 
423

424

425

426

427

428

429

430

431

432

433

434

435

436

437 scores for predicting dysphagia in critically ill patients after cardiac surgery. BMC Anesthesiol 19:7. 10.1186/s12871-019-0680-3

27

\section{Figure legends}

4

(1)

Figure 1. The nomogram used to predict PPCs in patients following thoracoscopic surgery.

37 The nomogram used to predict PPCs was created based on seven independent prognostic factors of the training cohort: ASA grade, duration of operation, duration of one-lung ventilation, and history of stroke, heart disease, COPD, and smoking. To use this nomogram, each variable position on the corresponding axis and the number should be found, and then the points of all variables are added. A line is drawn from the total points axis at the bottom line of the nomogram to determine the risk of PPCs. PPCs, postoperative pulmonary complications; ASA, American 
443 Society of Anesthesiologists; COPD, chronic obstructive pulmonary disease.

444

445

446 Figure 2. AUCs of the nomogram used to predict PPCs following thoracoscopic surgery in the

447 training and validation cohorts.

448

449 The nomogram generated by the training cohort.

450 The data in brackets represent a 95\% confidence interval.

451 AUC, the area under the receiver operating characteristic curve.

452

453

Figure 3. Calibration plot comparing predicted and actual PPCs following thoracoscopic surgery in the training and validation cohorts.

The 100-sample bootstrapped calibration plot for the prediction of PPCs is shown. (A) Validation of the predictive performance of the nomogram in estimating the risk of PPCs in the training cohort. (B) Validation of the predictive performance of the nomogram in estimating the risk of 
460 PPCs in the validation cohort. PPCs, postoperative pulmonary complications.

461 
Figure 1

Figure 1

The nomogram used to predict PPCs in patients following thoracoscopic surgery.

Points

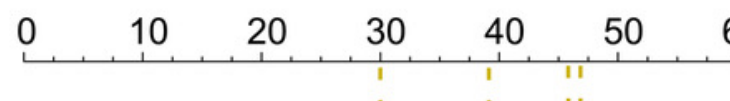

ASA grade

Duration of operation

(min)

Duration of one-lung ventilation ( $\mathrm{min})$

History of stroke

History of heart disease No

History of heart disease No

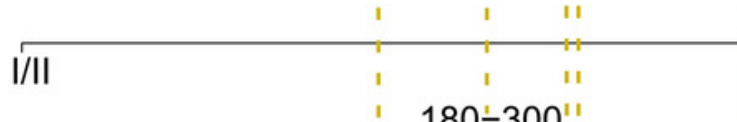

$\begin{array}{lllll}60 & 70 & 80 & 90 & 100\end{array}$

History of COPD

History of smoking

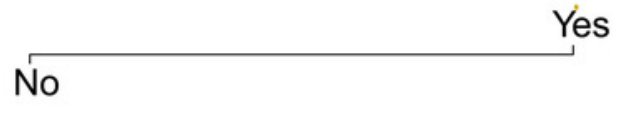

No

Yes

Total Points

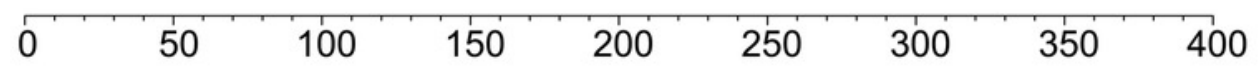

Risk of PPCs

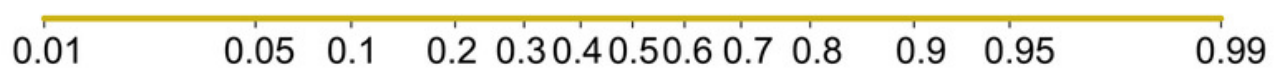


Figure 2

Figure 2

AUCs of the nomogram used to predict PPCs following thoracoscopic surgery in the training and validation cohorts.

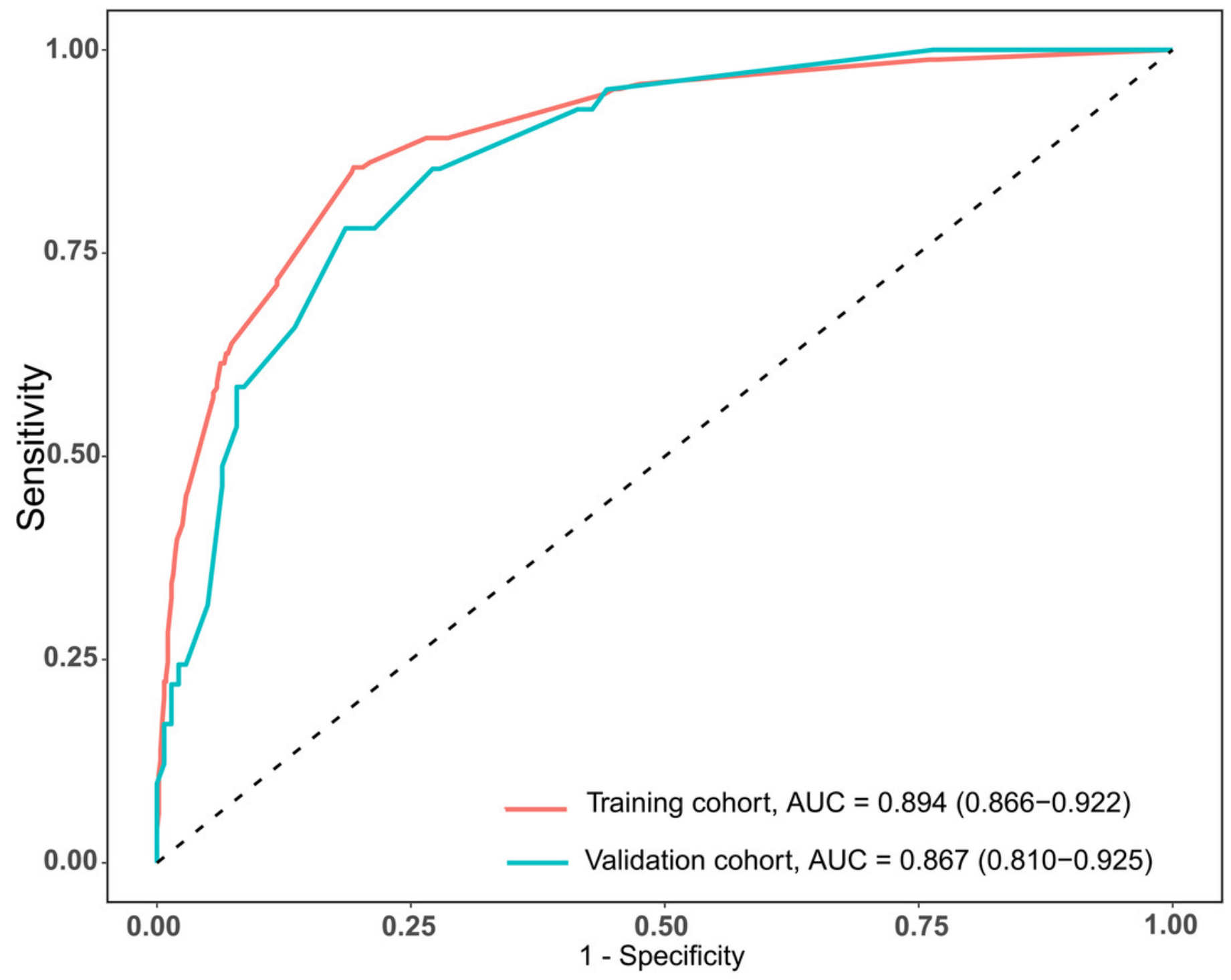


Figure 3

Figure 3

Calibration plot comparing predicted and actual PPCs following thoracoscopic surgery in the training and validation cohorts.
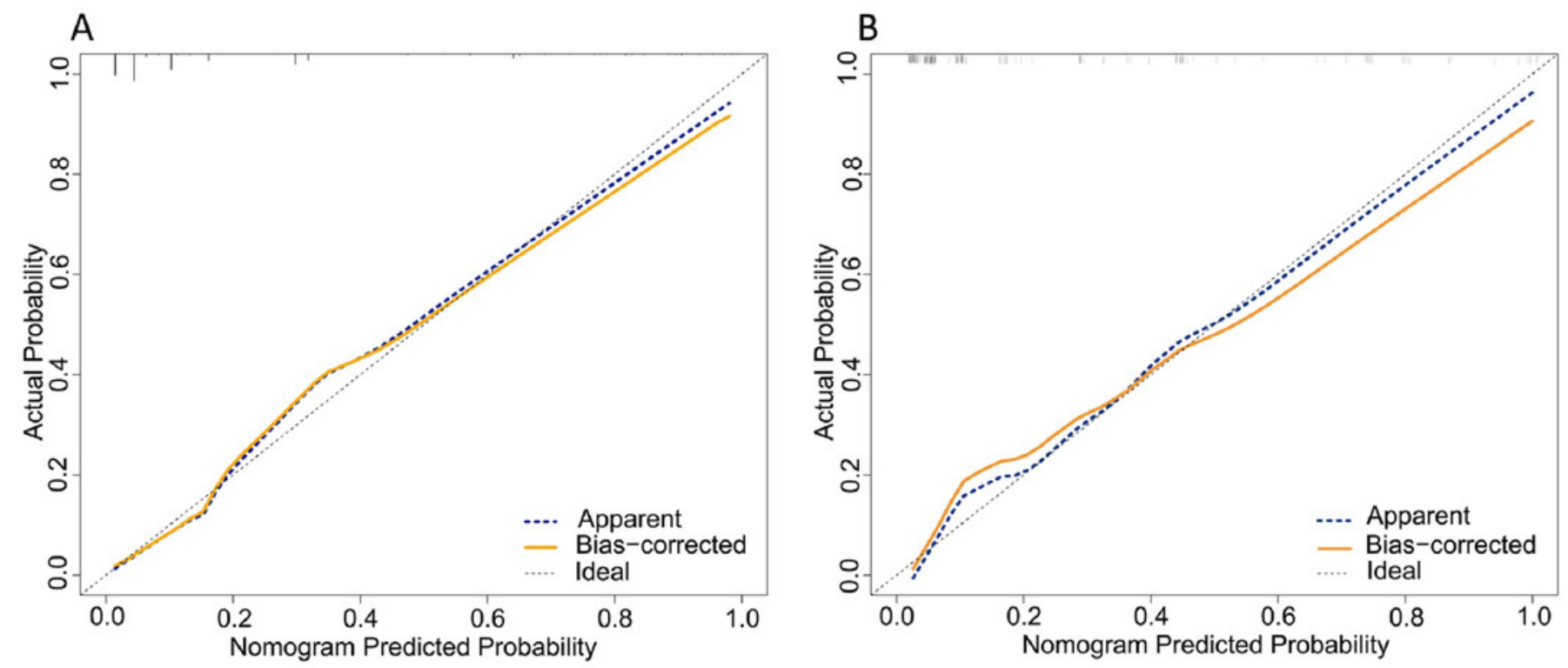


\section{Table $\mathbf{1}$ (on next page)}

Table 1

Descriptive variables of the patients following thoracoscopic surgery. 
1 Table 1. Descriptive variables of the patients following thoracoscopic surgery.

\begin{tabular}{|c|c|c|c|c|c|}
\hline$\square$ & $\begin{array}{l}\text { Total } \\
(n=905)\end{array}$ & $\begin{array}{l}\text { Training cohort } \\
\qquad(n=724)\end{array}$ & $\begin{array}{l}\text { Validation cohort } \\
\qquad(n=181)\end{array}$ & $p$-value & SMD \\
\hline Age (years) & & & & 0.670 & 0.130 \\
\hline$\geq 45$ & $159(17.57)$ & $133(18.37)$ & $26(14.36)$ & & \\
\hline $46-55$ & $196(21.66)$ & $155(21.41)$ & $41(22.65)$ & & \\
\hline $56-65$ & $230(25.41)$ & $186(25.69)$ & $44(24.31)$ & & \\
\hline $66-75$ & $256(28.29)$ & 199 (27.49) & $57(31.49)$ & & \\
\hline$>75$ & $64(7.07)$ & $51(7.04)$ & $13(7.18)$ & & \\
\hline Sex & & & & 0.514 & 0.145 \\
\hline Female & $331(36.57)$ & $261(36.05)$ & $70(38.67)$ & & \\
\hline Male & $574(63.43)$ & $463(63.95)$ & $111(61.33)$ & & \\
\hline ASA & & & & 0.789 & 0.022 \\
\hline I/II & $754(83.31)$ & $602(83.15)$ & $152(83.98)$ & & \\
\hline III/IV & $151(16.69)$ & $122(16.85)$ & $29(16.02)$ & & \\
\hline MAP (mmHg) & & & & 0.507 & 0.104 \\
\hline 70-105 & $510(56.35)$ & $403(55.66)$ & $107(59.12)$ & & \\
\hline$<70$ & $11(1.22)$ & $10(1.38)$ & $1(0.55)$ & & \\
\hline$>105$ & $384(42.43)$ & $311(42.96)$ & $73(40.33)$ & & \\
\hline Duration of operation (min) & & & & 0.424 & 0.112 \\
\hline$<180$ & $545(60.22)$ & $431(59.53)$ & $114(62.98)$ & & \\
\hline $180-300$ & $211(23.31)$ & $168(23.20)$ & $43(23.76)$ & & \\
\hline$>300$ & $149(16.46)$ & $125(17.27)$ & $24(13.26)$ & & \\
\hline Airway management & & & & 0.259 & 0.168 \\
\hline single-lumen & $29(3.20)$ & $21(2.90)$ & $8(4.42)$ & & \\
\hline double-lumen & $539(59.56)$ & $423(58.43)$ & $116(64.09)$ & & \\
\hline bronchial occluder & $328(36.24)$ & $272(37.57)$ & $56(30.94)$ & & \\
\hline LMA & $9(0.99)$ & $8(1.10)$ & $1(0.55)$ & & \\
\hline $\begin{array}{l}\text { Duration of one-lung ventilation } \\
\text { (min) }\end{array}$ & & & & 0.969 & 0.003 \\
\hline$\leq 60$ & $214(23.65)$ & $171(23.62)$ & $43(23.76)$ & & \\
\hline$>60$ & $691(76.35)$ & $553(76.38)$ & $138(76.24)$ & & \\
\hline BMI $\left(\mathrm{kg} / \mathrm{m}^{2}\right)$ & & & & 0.948 & 0.050 \\
\hline$\leq 18.5$ & $500(55.25)$ & $401(55.39)$ & 99 (54.70) & & \\
\hline $18.5-24$ & $123(13.59)$ & $100(13.81)$ & $23(12.71)$ & & \\
\hline $24-28$ & $226(24.97)$ & $178(24.59)$ & $48(26.52)$ & & \\
\hline$\geq 28$ & $56(6.19)$ & $45(6.22)$ & $11(6.08)$ & & \\
\hline History of hypertension & & & & 0.438 & 0.064 \\
\hline
\end{tabular}


No

Yes

History of diabetes mellitus

No

Yes

History of stroke

No

Yes

History of heart disease

No

Yes

History of COPD

No

Yes

History of smoking

No

Yes

History of alcohol use

No

Yes

Leukocyte counts $\left(\times 10^{9} / \mathrm{L}\right)$

4-10

$<4$

$>10$

Red blood cell counts $\left(\times 10^{12} / \mathrm{L}\right)$

Normal

Low

High

Platelet counts $\left(\times 10^{9} / \mathrm{L}\right)$

100-300

$<100$

$>300$

Aspartate aminotransferase (U/L)

$<40$

$\geq 40$

Alanine aminotransferase (U/L) $\begin{array}{lll}685(75.69) & 552(76.24) & 133(73.48) \\ 220(24.31) & 172(23.76) & 48(26.52)\end{array}$

$0.467 \quad 0.058$

855 (94.48)

686 (94.75)

169 (93.37)

50 (5.52)

38 (5.25)

12 (6.63)

859 (94.92)

691 (95.44)

0.151

0.112

46 (5.08)

33 (4.56)

168 (92.82)

13 (7.18)

$0.461 \quad 0.063$

833 (92.04)

664 (91.71)

169 (93.37)

72 (7.96)

60 (8.29)

12 (6.63)

857 (94.70)

681 (94.06)

0.088

0.156

176 (97.24)

$48(5.30)$

43 (5.94)

5 (2.76)

687 (75.91)

550 (75.97)

218 (24.09)

174 (24.03)

$0.659 \quad 0.036$

856 (94.59)

686 (94.75)

38 (5.25)

0.146

0.151

768 (84.86)

621 (85.77)

147 (81.22)

99 (10.94)

77 (10.64)

22 (12.15)

38 (4.20)

26 (3.59)

12 (6.63)

$0.321 \quad 0.122$

$\begin{array}{cc}750(82.87) & 598(82.60) \\ 127(14.03) & 106(14.64) \\ 28(3.09) & 20(2.76)\end{array}$

152 (83.98)

21 (11.60)

$8(4.42)$

$0.273 \quad 0.126$

$\begin{array}{ccc}811(89.61) & 654(90.33) & 157(86.74) \\ 33(3.65) & 26(3.59) & 7(3.87) \\ 61(6.74) & 44(6.08) & 17(9.39)\end{array}$

$0.476 \quad 0.058$

841 (92.93)

675 (93.23)

166 (91.71)

64 (7.07)

49 (6.77)

15 (8.29)

$0.777 \quad 0.049$ 


\begin{tabular}{|c|c|c|c|c|c|}
\hline$<50$ & 885 (97.79) & 707 (97.65) & 178 (98.34) & & \\
\hline$\geq 50$ & $20(2.21)$ & $17(2.35)$ & $3(1.66)$ & & \\
\hline Blood glucose (mmol/L) & & & & 0.454 & 0.064 \\
\hline Normal & 790 (87.29) & $629(86.88)$ & 161 (88.95) & & \\
\hline Abnormal & $115(12.71)$ & $95(13.12)$ & $20(11.05)$ & & \\
\hline Blood creatinine ( $\mu \mathrm{mol} / \mathrm{L})$ & & & & 0.745 & 0.058 \\
\hline$<111$ & $890(98.34)$ & $711(98.20)$ & $179(98.90)$ & & \\
\hline$\geq 111$ & $15(1.66)$ & $13(1.80)$ & $2(1.10)$ & & \\
\hline Blood urea nitrogen $(\mathrm{mmol} / \mathrm{L})$ & & & & 0.958 & 0.027 \\
\hline$<9.5$ & $882(97.46)$ & $705(97.38)$ & $177(97.79)$ & & \\
\hline$\geq 9.5$ & $23(2.54)$ & $19(2.62)$ & $4(2.21)$ & & \\
\hline Serum sodium (mmol/L) & & & & 0.935 & 0.030 \\
\hline $137-147$ & $863(95.36)$ & $691(95.44)$ & $172(95.03)$ & & \\
\hline$<137$ & $25(2.76)$ & $20(2.76)$ & $5(2.76)$ & & \\
\hline$>147$ & $17(1.88)$ & $13(1.80)$ & $4(2.21)$ & & \\
\hline Serum potassium (mmol/L) & & & & 0.635 & 0.039 \\
\hline Normal & $847(93.59)$ & $679(93.78)$ & $168(92.82)$ & & \\
\hline Abnormal & $58(6.41)$ & $45(6.22)$ & $13(7.18)$ & & \\
\hline Serum albumin $(g / L)$ & & & & 0.245 & 0.097 \\
\hline Normal & $445(49.17)$ & $349(48.20)$ & $96(53.04)$ & & \\
\hline Low & $460(50.83)$ & $375(51.80)$ & $85(46.96)$ & & \\
\hline Types of operation & & & & 0.510 & 0.223 \\
\hline Trans-thoracic vagotomy & $16(1.77)$ & $12(1.66)$ & $4(2.21)$ & & \\
\hline Wedge resection & $111(12.27)$ & $86(11.88)$ & $25(13.81)$ & & \\
\hline Segmental resection & $161(17.79)$ & $122(16.85)$ & $39(21.55)$ & & \\
\hline Lobectomy & $295(32.60)$ & $242(33.43)$ & $53(29.28)$ & & \\
\hline Total pneumonectomy & $7(0.77)$ & $5(0.69)$ & $2(1.10)$ & & \\
\hline Partial bilateral pneumonectomy & $25(2.76)$ & $22(3.04)$ & $3(1.66)$ & & \\
\hline Mediastinal surgery & $63(6.96)$ & $49(6.77)$ & $14(7.73)$ & & \\
\hline Oesophageal surgery & $207(22.87)$ & $172(23.76)$ & $35(19.34)$ & & \\
\hline Others & $20(2.21)$ & $14(1.93)$ & $6(3.31)$ & & \\
\hline PPCs & & & & 0.937 & 0.007 \\
\hline No & $698(77.13)$ & $558(77.07)$ & $140(77.35)$ & & \\
\hline Yes & $207(22.87)$ & $166(22.93)$ & $41(22.65)$ & $\square$ & $\square$ \\
\hline
\end{tabular}

2 Values are presented as counts.

3 Blood biochemical data were routine examination results before the operation. 
4 SMD, standardized mean difference; PPCs, postoperative pulmonary complications; ASA,

5 American Society of Anesthesiologists; MAP, mean arterial pressure; LMA, laryngeal mask airway;

6 COPD, chronic obstructive pulmonary disease. 


\section{Table 2 (on next page)}

Table 2

Univariate and multivariable logistic regression analyses of variables for PPCs after thoracoscopic surgery in the training cohort. 
1 Table 2. Univariate and multivariable logistic regression analyses of variables for PPCs after 2 thoracoscopic surgery in the training cohort.

\begin{tabular}{|c|c|c|c|c|c|}
\hline \multirow[t]{2}{*}{ Project } & \multirow[b]{2}{*}{$\square$} & \multicolumn{2}{|c|}{ Univariate analysis } & \multicolumn{2}{|c|}{ Multivariate analysis } \\
\hline & & OR $(95 \% \mathrm{Cl})$ & $p$-value & OR $(95 \% \mathrm{Cl})$ & $p$-value \\
\hline \multirow[t]{5}{*}{ Age (years) } & $\leq 45$ & Ref & & & \\
\hline & $46-55$ & $2.515(1.066-6.622)$ & 0.045 & & \\
\hline & $56-65$ & $5.250(2.416-13.155)$ & $<0.001$ & & \\
\hline & $66-75$ & $10.428(4.930-25.704)$ & $<0.001$ & & \\
\hline & $>75$ & $17.309(7.097-47.375)$ & $<0.001$ & & \\
\hline \multirow[t]{2}{*}{ Sex } & female & Ref & & & \\
\hline & male & $1.782(1.215-2.653)$ & 0.004 & & \\
\hline \multirow[t]{2}{*}{ ASA } & $1 / I I$ & Ref & & Ref & \\
\hline & III/IV & $14.776(9.485-23.437)$ & $<0.001$ & $10.171(5.598-18.926)$ & $<0.001$ \\
\hline \multirow[t]{3}{*}{ MAP (mmHg) } & $70-105$ & Ref & & & \\
\hline & $<70$ & $0.404(0.022-2.190)$ & 0.392 & & \\
\hline & $>105$ & $1.216(0.857-1.724)$ & 0.273 & & \\
\hline \multirow[t]{3}{*}{ Duration of operation (min) } & $<180$ & Ref & & Ref & \\
\hline & $180-300$ & $3.229(2.073-5.037)$ & $<0.001$ & $2.480(1.363-4.563)$ & 0.003 \\
\hline & $>300$ & $8.524(5.414-13.563)$ & $<0.001$ & $12.516(5.232-17.766)$ & $<0.001$ \\
\hline \multirow[t]{4}{*}{ Airway management } & Bronchial occluder & Ref & & & \\
\hline & double-lumen & $1.335(0.438-5.800)$ & 0.650 & & \\
\hline & LMA & $2.774(0.909-12.067)$ & 0.109 & & \\
\hline & single-lumen & $0.000(0.000-0.000)$ & 0.979 & & \\
\hline \multicolumn{6}{|l|}{ Duration of one-lung } \\
\hline \multirow[t]{2}{*}{ ventilation (min) } & $\leq 60$ & Ret & & Ret & \\
\hline & $>60$ & $1.000(1.000-1.000)$ & $<0.001$ & $2.947(1.257-7.648)$ & 0.018 \\
\hline \multirow[t]{4}{*}{ BMI $\left(\mathrm{kg} / \mathrm{m}^{2}\right)$} & $<18.5$ & Ref & & & \\
\hline & $18.5-24$ & $0.625(0.344-1.080)$ & 0.105 & & \\
\hline & $24-28$ & $0.942(0.619-1.417)$ & 0.777 & & \\
\hline & $\geq 28$ & $1.516(0.277-1.395)$ & 0.306 & & \\
\hline \multirow[t]{2}{*}{ History of hypertension } & No & Ref & & & \\
\hline & Yes & $1.481(0.999-2.177)$ & 0.048 & & \\
\hline \multirow[t]{2}{*}{ History of diabetes mellitus } & No & Ref & & & \\
\hline & Yes & $1.812(0.880-3.567)$ & 0.093 & & \\
\hline \multirow[t]{2}{*}{ History of stroke } & No & Ref & & Ref & \\
\hline & Yes & $5.022(2.472-10.442)$ & $<0.001$ & $4.209(1.518-11.683)$ & 0.006 \\
\hline
\end{tabular}




\begin{tabular}{|c|c|c|c|c|c|}
\hline \multirow[t]{2}{*}{ History of heart disease } & No & Ref & \multicolumn{3}{|c|}{ Ref } \\
\hline & Yes & $7.233(4.171-12.832)$ & $<0.001$ & $2.001(0.901-4.479)$ & 0.089 \\
\hline \multirow[t]{2}{*}{ History of COPD } & No & Ref & & Ref & \\
\hline & Yes & $7.345(3.871-14.467)$ & $<0.001$ & $2.888(1.230-6.980)$ & 0.016 \\
\hline \multirow[t]{2}{*}{ History of smoking } & No & Ref & & Ref & \\
\hline & Yes & $5.962(4.083-8.753)$ & $<0.001$ & $4.101(2.544-6.654)$ & $<0.001$ \\
\hline \multirow[t]{2}{*}{ History of alcohol use } & No & Ref & & & \\
\hline & Yes & $1.594(0.760-3.168)$ & 0.196 & & \\
\hline \multirow[t]{3}{*}{ Leukocyte counts (× 109/L) } & $4-10$ & Ref & & & \\
\hline & $<4$ & $1.325(0.760-2.234)$ & 0.304 & & \\
\hline & $>10$ & $1.570(0.633-3.577)$ & 0.301 & & \\
\hline \multirow[t]{3}{*}{ Red blood cell counts $\left(\times 10^{12} / \mathrm{L}\right)$} & Normal & Ref & & & \\
\hline & Low & $1.828(1.157-2.850)$ & 0.009 & & \\
\hline & Hight & $0.927(0.262-2.580)$ & 0.894 & & \\
\hline \multirow[t]{3}{*}{ Platelet counts $\left(\times 10^{9} / \mathrm{L}\right)$} & $100-300$ & Ref & & & \\
\hline & $<100$ & $1.227(0.472-2.852)$ & 0.650 & & \\
\hline & $>300$ & $0.740(0.314-1.548)$ & 0.454 & & \\
\hline \multicolumn{2}{|l|}{ Aspartate aminotransferase } & Ref & & & \\
\hline \multirow[t]{2}{*}{ (U/L) } & $<40$ & & & & \\
\hline & $\geq 40$ & $0.971(0.463-1.883)$ & 0.934 & & \\
\hline \multicolumn{2}{|l|}{ Alanine aminotransferase } & Rof & & & \\
\hline \multirow[t]{2}{*}{ (U/L) } & $<50$ & net & & & \\
\hline & $\geq 50$ & $1.413(0.444-3.871)$ & 0.522 & & \\
\hline \multirow[t]{2}{*}{ Blood glucose (mmol/L) } & Normal & Ref & & & \\
\hline & Abnormal & $0.882(0.509-1.468)$ & 0.641 & & \\
\hline \multirow[t]{2}{*}{ Blood creatinine $(\mu \mathrm{mol} / \mathrm{L})$} & $<111$ & Ref & & & \\
\hline & $\geq 111$ & $2.135(0.638-6.489)$ & 0.189 & & \\
\hline \multirow[t]{2}{*}{ Blood urea nitrogen (mmol/L) } & $<9.5$ & Ref & & & \\
\hline & $\geq 9.5$ & $1.572(0.545-4.047)$ & 0.367 & & \\
\hline \multirow[t]{3}{*}{ Serum sodium (mmol/L) } & $137-147$ & Ref & & & \\
\hline & $<137$ & $1.143(0.367-3.003)$ & 0.799 & & \\
\hline & $>147$ & $2.143(0.640-6.518)$ & 0.187 & & \\
\hline Serum potassium (mmol/L) & Normal & Ref & & & \\
\hline
\end{tabular}




\begin{tabular}{llcc} 
& Abnormal & $1.566(0.790-2.964)$ & 0.181 \\
Serum albumin (g/L) & Normal & Ref & \\
& Low & $1.611(1.134-2.300)$ & 0.008 \\
Types of operation & Wege resection & Ref & \\
& Trans-thoracic vagotomy & $0.000(0.000-0.000)$ & 0.983 \\
& Segmental resection & $1.180(0.533-2.717)$ & 0.687 \\
& Lobectomy & $1.687(0.859-3.578)$ & 0.147 \\
& Total pneumonectomy & $0.000(0.000$, inf $)$ & 0.989 \\
& Partial bilateral & & \\
& pneumonectomy & $2.005(0.571-6.333)$ & 0.248 \\
& Mediastinal surgery & $0.290(0.044-1.142)$ & 0.118 \\
& Esophageal surgery & $6.069(3.122-12.808)$ & $<0.001$ \\
& Others & $0.524(0.028-3.062)$ & 0.553 \\
\hline
\end{tabular}

3 Blood laboratory data were routine examination results before the operation.

4 OR, odds ratio; $\mathrm{Cl}$, confidence interval; ASA, American Society of Anesthesiologists; MAP, mean

5 arterial pressure; LMA, laryngeal mask airway; COPD, chronic obstructive pulmonary disease. 


\section{Table 3 (on next page)}

Table 3

Prediction performance of the nomogram in the training cohort and the validation cohort. 
1 Table 3. Prediction performance of the nomogram in the training cohort and the validation

2 cohort.

\begin{tabular}{ccc}
\hline & $\begin{array}{c}\text { Training cohort } \\
(\boldsymbol{n}=\mathbf{7 2 4})\end{array}$ & $\begin{array}{c}\text { Validation cohort } \\
(\boldsymbol{n}=\mathbf{1 8 1})\end{array}$ \\
\hline PPCs & $166(22.93 \%)$ & $41(22.65 \%)$ \\
\hline Nomogram points (Mean士SD) & $101.27 \pm 84.78$ & $96.55 \pm 78.54$ \\
Nomogram points (Min, Max) & $(0,397)$ & $(0,305)$ \\
cut-off points & 134 & 134 \\
Accuracy & 0.817 & 0.807 \\
Precision & 0.567 & 0.552 \\
Sensitivity & 0.855 & 0.814 \\
Specificity & 0.806 & 0.780 \\
AUC of nomogram (95\% Cl) & $0.894(0.866-0.922)$ & $0.867(0.810-0.925)$ \\
C-index of nomogram (95\% Cl) & $0.894(0.866-0.921)$ & $0.868(0.811-0.925)$ \\
\hline
\end{tabular}

3 The nomogram generated by the training cohort.

4 PPCs, postoperative pulmonary complications; SD, standard deviation; AUC, area under the

5 receiver operating characteristic curve; $\mathrm{Cl}$, confidence interval.

6

7

8

9

10

11

12 
